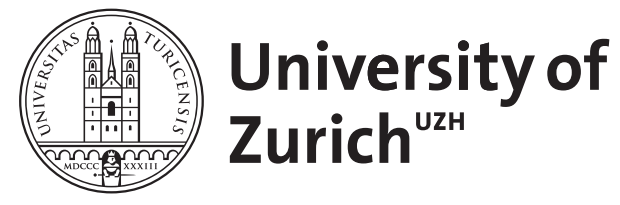
Archive

University of Zurich

University Library

Strickhofstrasse 39

CH-8057 Zurich

www.zora.uzh.ch

Year: 2008

Bile aspiration for diagnosis of Candida glabrata cholecystitis

Misselwitz, B ; Imhof, A ; Schneemann, M

DOI: https://doi.org/10.1007/s15010-007-7261-y

Posted at the Zurich Open Repository and Archive, University of Zurich

ZORA URL: https://doi.org/10.5167/uzh-17205

Journal Article

Published Version

Originally published at:

Misselwitz, B; Imhof, A; Schneemann, M (2008). Bile aspiration for diagnosis of Candida glabrata cholecystitis. Infection, 36(2):189-190.

DOI: https://doi.org/10.1007/s15010-007-7261-y 


\section{Bile Aspiration for Diagnosis of Candida glabrata Cholecystitis}

Infection with Candida species is a common nosocomial problem in critically ill patients [1-3]. Candidemia is associated with a high mortality rate; the clinical outcome strongly depends on timely application of an appropriate antifungal [4]. However, even though many antifungals are available now, clinical and microbiological failures of antifungal therapy are encountered frequently [3]. Little evidence exists to guide the management of this difficult situation. The change of antifungal therapy to an alternative agent is often considered. However, failure of antifungal therapy might also be due to a second fungus missed at the time of the initial diagnosis. Therefore, continuous search for an infectious focus should be a priority. We report the case of a breakthrough infection due to Candida glabrata in a patient with Candida albicans candidemia. C. glabrata cholecystitis was diagnosed with bile aspiration which also at least partially cured the infectious nidus.

A 76-year-old woman was referred to the ICU of our hospital with severe $C$. albicans sepsis. The personal history of the patient was remarkable for surgical removal of the bladder because of a carcinoma. Local complications prompted further operations, including construction of an ileocoecal reservoir. On day 4 of the hospitalization an ileum conduit was connected to the reservoir. The postoperative course was complicated by peritonitis due to a leaky anastomosis, making an ileostoma necessary on day 20. Because of short bowel syndrome parenteral nutrition was started. The patient slowly improved over the next weeks.

On the 11th week of the hospitalization the patient was transferred to the ICU with dyspnea and delirium. Computer tomography (CT) of the thorax on day 75 showed pulmonary embolism and infiltrates, prompting anticoagulation and antibiotic treatment. Two blood cultures (BacTAlert, bioMérieux, Marcy l'Etoile, France) grew $C$. albicans after 5 days. The tip of the central catheter also grew $C$. albicans. CHROMagar Candida plates (CHROMagar Company, Paris, France) were used for primary identification. The diagnosis was confirmed using API Candida (bioMérieux). Antifungal therapy with fluconazole, $400 \mathrm{mg}$ iv once daily, was initiated. Despite persistently elevated markers for systemic inflammation, further blood cultures were negative.

On day 90 the CT-scan demonstrated new peribronchovascular infiltrates, suggestive of Aspergillus pneu- monia. The antifungal regiment was changed to voriconazole, $200 \mathrm{mg}$ iv twice daily. Abdominal complaints prompted a CT-scan of the abdomen on day 95, demonstrating an enlarged gall bladder with perivesicular fluid, suggestive of acalculous cholecystitis. On day 97, $150 \mathrm{ml}$ of slightly turbid gall bladder fluid were aspirated. Semiquantitative cultures grew moderate amounts of $C$. glabrata (no bacterial growth was observed). The susceptibility testing of $C$. glabrata revealed intermediate susceptibility to fluconazole (minimal inhibitory concentration, MIC, $16 \mu \mathrm{g} / \mathrm{ml}$ ) but susceptibility to voriconazole (MIC $0.25 \mu \mathrm{g} / \mathrm{ml}$ ). On day 111 the patient had substantially improved and was transferred to a peripheral hospital. Within the following weeks, short bowel syndrome prevented complete recovery. Surgery was attempted to correct the ileostoma. The patient, however, succumbed to postoperative septic complications. Blood cultures did not show resurgence of candidemia.

Candida species account for $2.9 \%$ of bloodstream pathogens in Swiss hospitals [1]. Although Candida species are considered to be normal commensal organisms, disseminated candidiasis is life threatening. Common risk factors include colonization, broad-spectrum antibiotics, a central venous catheter, parenteral nutrition, gastrointestinal or cardiac surgery, prolonged hospital or ICU stay, burns, diabetes mellitus, and immunosuppression [3]. Most of these conditions favor an overgrowth of yeast in the bowel, provide an entrance route or can be considered a marker of severe underlying disease. Remarkably, our patient fulfilled all these risk factors, except no burn injuries and immunosuppression were present.

The most common Candida species involved is $C$. albicans, accounting for $66 \%$ of Candida isolates in Swiss hospitals, followed by $C$. glabrata $(15 \%)$ and $C$. tropicalis $(2-10 \%)$ [1]. Our patient has been at high risk for $C$. glabrata infection. According to a recent study, certain

Infection 2008; 36: 189-190

DOI 10.1007/s15010-007-7261-Y

\footnotetext{
B. Misselwitz, A. Imhof, M. Schneemann (corresponding author) Dept. of Medicine, University Hospital Zurich, Raemistrasse 100, CH-8o91, Zürich, Switzerland; Phone: (+41/44) 255-1111, Fax: -4445, e-mail: markus.schneemann@usz.ch
}

Received: June 11, $2007 \cdot$ Revision accepted: September 19, 2007 Published online: March 10, 2008 
broad spectrum antibiotics and pretreatment with azoleantifungal agents favor infection with $C$. glabrata or Candida krusei over C. albicans. The risk was strongest with two of the antibiotics our patient had been exposed to: piperacillin/tazobactam and vancomycin, which increase the risk by a factor of 4.2 or 6.5 , respectively [5].

The differential diagnosis of an enlarged gall bladder in an ICU patient is challenging. On the one hand, hydrops frequently develops in patients who cannot be fed orally and resolves without specific treatment. On the other, infectious cholecystitis requires emergent surgical or percutaneous treatment. In a recent study, commonly used biochemical or radiological criteria such as ASAT, ALAT, AP, GGT, gall bladder wall enlargement, or pericholecystic fluid failed to predict subsequent isolation of microorganisms in the gall aspirate [6]. Therefore, the suspicion of infectious cholecystitis largely depends on clinical judgment. In a patient like ours with sepsis and no other focus, a suspicious gall bladder should be further evaluated. In our case, unequivocal microbiological evidence of gall bladder infection has been provided, since $C$. glabrata was isolated from the bile aspirate. This is highly unusual because Candida species are an infrequent gall bladder pathogen with $C$. albicans responsible for the majority of cases and C. glabrata cholecystitis only anecdotically reported $[7,8]$.

The first-line empirical antifungal for candidemia in hemodynamically stable, non-neutropenic patients and the first-line treatment of C. albicans is fluconazole. As soon as species differentiation reveals $C$. krusei or C. glabrata fluconazole needs to be replaced, since virtually all $C$. krusei and $13-75 \%$ of C. glabrata isolates are fluconazole resistant $[3,9,10]$. The therapeutic options now include amphothericin B, caspofungin, or voriconazole. While voriconazole is clearly more effective than fluconazole against C. krusei or C. glabrata, its use against both pathogens remains controversial [2, 3, 11, 12]. For instance, breakthrough infections with $C$. glabrata under voriconazole prophylaxis were noted; however, the minimal inhibitory concentration of the isolates has been consistently above the proposed break point of $>1 \mu \mathrm{g} / \mathrm{ml}$ [13]. In our case the minimal inhibitory concentration for voriconazole $(0.25 \mu \mathrm{g} / \mathrm{ml})$ remained well below this break point, making continued treatment with this drug a reasonable option.

However, even though several potent antifungals are available, success of antifungal treatment is uncertain. Therefore, local therapy might be an attractive option. In our case percutaneous cholecystostomy not only demonstrated the double yeast infection but also in addition at least partially cured the local infection. Recently, a study compared percutaneous cholecystostomy with surgery in high-risk patients with acute cholecystitis, demonstrating a low rate of complications [14].
In summary, failure of fluconazole to improve a critical state in a patient with $C$. albicans infection raises the possibility of another Candida species. A search for a possible focus should be attempted; in our case, aspiration of an enlarged gallbladder identified C. glabrata and at least partially cured the infectious focus. Gallbladder aspiration seems to be a safe and effective option in patients with suspected biliary candidiasis.

\section{B. Misselwitz, A. Imhof, M. Schneemann}

\section{References}

1. Marchetti O, et al. Epidemiology of candidemia in Swiss tertiary care hospitals: secular trends, 1991-2000. Clin Infect Dis 2004; 38: 311-320.

2. Fluckiger $\mathrm{U}$, et al. Treatment options of invasive fungal infections in adults. Swiss Med Wkly 2006; 136: 447-463.

3. Spellberg BJ, Filler SG, Edwards JE Jr: Current treatment strategies for disseminated candidiasis. Clin Infect Dis 2006; 42: 244-251.

4. Blot SI, et al. Effects of nosocomial candidemia on outcomes of critically ill patients. Am J Med 2002; 113: 480-485.

5. Lin MY, et al. Prior antimicrobial therapy and risk for hospitalacquired Candida glabrata and Candida krusei fungemia: a case-case-control study. Antimicrob Agents Chemother 2005; 49: 4555-456o.

6. Beardsley SL, et al. Predicting infected bile among patients undergoing percutaneous cholecystostomy. Cardiovasc Intervent Radiol 2005; 28: 319-325.

7. Warren GH, Marsh S: Granulomatous Torulopsis glabrata cholecystitis in a diabetic. Am J Clin Pathol 1982; 78: 406-410.

8. Miller DD: Postoperative acalculous cholecystitis due to Torulopsis glabrata. Arch Surg 1976; 111: 1404-1405.

9. Drago $M$, Scaltrito MM, Morace G: In vitro activity of voriconazole and other antifungal agents against clinical isolates of Candida glabrata and Candida krusei. Eur J Clin Microbiol Infect Dis 2004; 23: 619-624.

10. St-Germain $\mathrm{G}$, et al. Prevalence and antifungal susceptibility of 442 Candida isolates from blood and other normally sterile sites: results of a 2-year (1996 to 1998) multicenter surveillance study in Quebec, Canada. J Clin Microbiol 2001; 39: 949-953.

11. Kullberg BJ, et al. Voriconazole versus a regimen of amphotericin B followed by fluconazole for candidaemia in non-neutropenic patients: a randomised non-inferiority trial. Lancet 2005; 366: 1435-1442.

12. Ostrosky-Zeichner $L$, et al. Voriconazole salvage treatment of invasive candidiasis. Eur J Clin Microbiol Infect Dis 2003; 22: 651-655.

13. Imhof A, et al. Breakthrough fungal infections in stem cell transplant recipients receiving voriconazole. Clin Infect Dis 2004; 39: 743-746.

14. Hatzidakis AA, et al. Acute cholecystitis in high-risk patients: percutaneous cholecystostomy vs conservative treatment. Eur Radiol 2002; 12: 1778-1784. 